\title{
Physiological Correlates of Neurobehavioral Disinhibition that Relate to Drug Use and Risky Sexual Behavior in Adolescents with Prenatal Substance Exposure
}

\author{
Elisabeth Conradt ${ }^{a, b}$ Linda L. Lagasse ${ }^{a-c}$ Seetha Shankaran ${ }^{d}$ Henrietta Bada ${ }^{e}$ \\ Charles R. Bauer $^{f}$ Toni M. Whitaker ${ }^{\text {g J Jane A. Hammond }}{ }^{\text {h }}$ Barry M. Lester ${ }^{\text {a-c }}$ \\ ${ }^{a}$ Brown Center for the Study of Children at Risk, Department of Pediatrics, Women and Infants Hospital of Rhode \\ Island, and Departments of ${ }^{\mathrm{b}}$ Psychiatry and ' Pediatrics, Warren Alpert Medical School of Brown University, \\ Providence, R.I., d Department of Pediatrics, Wayne State University School of Medicine, Detroit, Mich., ${ }^{e}$ Department \\ of Pediatrics, University of Kentucky College of Medicine, Lexington, Ky., fDepartment of Pediatrics, University of \\ Miami, Miller School of Medicine, Miami, Fla., ${ }^{9}$ Department of Pediatrics, University of Tennessee, Memphis, Tenn., \\ and hesearch Triangle Institute, Research Triangle Park, N.C., USA
}

\section{Key Words}

Cortisol - Heart rate variability - Adversity - Sex differences .

Teenage substance use onset - Behavioral problems .

Executive function

\begin{abstract}
Physiological correlates of behavioral and emotional problems, substance use onset and initiation of risky sexual behavior have not been studied in adolescents with prenatal drug exposure. We studied the concordance between baseline respiratory sinus arrhythmia (RSA) at age 3 and baseline cortisol levels at age 11 . We hypothesized that children who showed concordance between RSA and cortisol would have lower neurobehavioral disinhibition scores which would in turn predict age of substance use onset and first sexual intercourse. The sample included 860 children aged 16 years participating in the Maternal Lifestyle Study, a multisite longitudinal study of children with prenatal exposure to cocaine and other substances. Structural equation modeling was used to test pathways between prenatal substance ex-
\end{abstract}

posure, early adversity, baseline RSA, baseline cortisol, neurobehavioral disinhibition, drug use, and sexual behavior outcomes. Concordance was studied by examining separate male and female models in which there were statistically significant interactions between baseline RSA and cortisol. Prenatal substance exposure was operationalized as the number of substances to which the child was exposed. An adversity score was computed based on caregiver postnatal substance use, depression and psychological distress, number of caregiver changes, socioeconomic and poverty status, quality of the home environment, and child history of protective service involvement, abuse and neglect. RSA and cortisol were measured during a baseline period prior to the beginning of a task. Neurobehavioral disinhibition, based on composite scores of behavioral dysregulation and executive dysfunction, substance use and sexual behavior were derived from questionnaires and cognitive tests administered to the child. Findings were sex specific. In females, those with discordance between RSA and cortisol (high RSA and low cortisol or low RSA and high cortisol) had the most executive dysfunction which, in turn, predicted earlier initiation of al-

\section{KARGER}

E-Mail karger@karger.com

www.karger.com/dne
(C) 2014 S. Karger AG, Base

$0378-5866 / 14 / 0364-0306 \$ 39.50 / 0$
Barry M. Lester, $\mathrm{PhD}$

Founding Director, Brown Center for the Study of Children at Risk, Warren Alpert Medical School of Brown University, Women and Infants Hospital of Rhode Island 101 Dudley Street, Providence, RI 02905 (USA)

E-Mail Barry_Lester@Brown.edu 
cohol by age 16 . Among boys, there also existed a significant baseline RSA by baseline cortisol interaction. Boys with low baseline RSA and high baseline cortisol had the highest levels of behavioral dysregulation. This increase in behavioral dysregulation was in turn related to initiation of alcohol use by age 16 and lower age of first sexual intercourse. We found sex-specific pathways to the initiation of alcohol use and risky sexual behavior through the combined activity of parasympathetic and neuroendocrine functioning. The study of multiple physiological systems may suggest new pathways to the study of age of onset of substance use and engagement in risky sexual behavior in adolescents.

(c) 2014 S. Karger AG, Basel

\section{Introduction}

Children with prenatal drug exposure are more likely to exhibit problems with behavioral and emotional regulation, initiate the use of substances and engage in risky sexual behavior earlier compared to their unexposed counterparts $[1,2]$. However, the underlying physiological processes that may be contributing to this increased risk of poor behavioral outcomes has not been well studied. Recent work highlights the importance of studying multiple physiological systems in order to achieve a more accurate picture of behavioral functioning compared to studying one system in isolation [3]. In this study we focused on the parasympathetic and neuroendocrine systems, giving evidence that both are predictive of behavioral regulation later in life [4-6].

Respiratory sinus arrhythmia (RSA) is an index of the parasympathetic nervous system and is a phenomenon whereby the heart rate shows high frequency variability in time with respiration [4]. High baseline RSA reflects the functional relationship between the central nervous system and the heart as mediated by the vagus $[7,8]$ and is related to neural integrity and readiness to respond to environmental stressors [4]. It may be an important predictor of behavioral and emotional functioning later in life [4, 9-11]. Cortisol is one of the hormonal products of the limbic-hypothalamic-pituitary-adrenocortical system. The neuroendocrine and parasympathetic systems do not operate in isolation [12]. Both the neuroendocrine and autonomic nervous systems are mediated by the limbic forebrain, hypothalamus and brainstem [13], thought of as the central autonomic network [14]. This network regulates the parasympathetic nervous system directly and HPA activity via the amygdala. Only one study that we know of has examined relations between baseline cortisol, baseline
RSA and measures of problem behavior in older children. In this study, children with both high baseline RSA and high baseline cortisol, or low baseline RSA and low baseline cortisol, had low levels of depressive symptoms in 1 of the 4 models tested, while in the other 3 models, children with high baseline RSA and high baseline cortisol had the lowest levels of depression and anxiety [12].

In previous work we found that prenatal cocaine exposure predicted neurobehavioral disinhibition in adolescents, which in turn was related to the initiation of substance use [1]. Neurobehavioral disinhibition is a singular multiproblem dimension involving lack of behavior control and poor emotional modulation that has features of disruptive behavior disorders, affective and anxiety disorders, cognitive impairment, and poor self-control. Here, we examine whether individual differences in parasympathetic and neuroendocrine functioning predict neurobehavioral disinhibition and, in turn, drug use and risky sexual behavior outcomes. Based on the existing literature, we hypothesize that children who exhibit concordance between their parasympathetic and neuroendocrine systems will have the lowest levels of neurobehavioral disinhibition and, in turn, will initiate the use of substances later and have a later onset of age of first sexual intercourse. Given that both drug use and risky sexual behavior outcomes may differ between the sexes $[15,16]$, we tested our models separately for males and females.

\section{Methods}

\section{Participants}

We used data from 1,388 participants drawn from the Maternal Lifestyle Study, a multisite investigation of the effects of prenatal substance exposure in a longitudinal follow-up from 1 month to 16 years. In the present study data were used from the birth visit and when children were aged 3, 11 and 13 years. Participants were recruited from Detroit, Mich., Memphis, Tenn., Miami, Fla., and Providence, R.I., USA. The Maternal Lifestyle Study sample includes children in the following racial and ethnic categories: African American (77\%), Caucasian (16\%), Hispanic (6\%), and children whose parents identified other racial or ethnic backgrounds (1\%). Details on the enrollment and exclusion criteria are described elsewhere [17]. In brief, the families were selected for the exposed group (maternal report of cocaine or opiate use during pregnancy or gas chromatography - mass spectrometry confirmation of presumptive positive meconium screens for cocaine or opiate metabolites) or the comparison group (maternal denial of cocaine or opiate use during pregnancy and a negative enzyme multiplied immunoassay meconium screen for cocaine and opiate metabolites). The exposed and comparison youths were group matched on race, gender and gestational age within each study site. Background substances associated with cocaine use (alcohol, tobacco and marijuana) were present in both groups; thus, most participants were polysubstance exposed. 
The study was approved by the institutional review board at each study site, and written informed consent (from caregivers) was obtained for all participants. Each site had a certificate of confidentiality from the National Institute on Drug Abuse (NIDA). Examiners were blinded to group status.

\section{Measures}

Polysubstance Exposure

Prenatal substance exposure was measured as a summative index ranging from 0 to 5 for use of cocaine, opiates, marijuana, alcohol and tobacco during pregnancy, as previously reported [18]. Maternal report/meconium screen of drug use $(1=$ yes, $0=$ no $)$ prenatally was computed; 1 point was assigned for each substance used. Dichotomous measures are frequently used in testing prenatal substance exposure effects $[18,19]$.

\section{Early Adversity}

Early adversity was a summative risk index assessment from birth to age 3 and included 9 risk factors. Each risk factor was either a continuous scale or a count score that was dichotomized to create an overall risk index $(0=$ no/none, $1=$ yes $/ 1$ or more).

Maternal Report of Postnatal Substance Use

Any maternal report of postnatal substance use of cocaine, opiates, tobacco, alcohol or marijuana up to the year 3 assessment was assessed using the Caretaker Inventory of Substance Use [20].

Chronic Poverty Status

Chronic poverty status was calculated as income below USD 10,000 for at least $75 \%$ of the visits.

Low Social Status

Low social status was scored from the Hollingshead Index of Social Position [21]. Education and occupation were averaged over annual visits. Participants received 1 point if their Index of Social Position score fell 1 standard deviation (SD) or more below the mean.

\section{Primary Caretaker Changes}

The presence of primary caretaker changes was assessed annually.

\section{Sexual or Physical Abuse or Neglect}

Caregivers were interviewed about whether or not there had been any reports of sexual or physical abuse and/or neglect annually from the birth to age 3 assessment.

\section{Caregiver Depression}

Children received 1 point on the cumulative risk index if assessments of caregiver depression were 1 SD or greater above the mean for averaged depressive symptoms on the caregiver-reported Beck Depression Inventory [22] at ages 4 months $(\alpha=0.88)$ and 2.5 years $(\alpha=0.89)$.

\section{Caregiver Psychological Distress}

This risk item was calculated as present if assessments of caregiver psychological distress were $1 \mathrm{SD}$ or greater above the mean for total psychological symptoms on the Brief Symptom Inventory [23] at ages 4 months $(\alpha=0.96)$ and 2.5 years $(\alpha=0.96)$.
Home Environment

This risk item was coded as present if scores fell $1 \mathrm{SD}$ or more below the mean on the Home Observation Measurement of the Environment [24] as assessed by a home visitor when the child was 10 months old $(\alpha=0.83)$.

History of Child Protective Services Involvement

If the child had any history of Child Protective Services involvement, assessed annually by caregiver report until the year 3 assessment, then 1 point was given on this risk index.

\section{Baseline RSA}

Baseline RSA was measured at age 3 because of research suggesting that RSA in early childhood is predictive of later behavioral problems $[25,26]$. RSA was derived from the R-R time series collected from digitized ECG recordings using Porges algorithm from MXEdit [27]. ECGs were recorded via three electrodes placed on the child's chest and abdomen. The ECG signal was sampled at $1 \mathrm{kHz}$ and stored on a computer for later scoring. Interbeat intervals were defined by detection of R-waves to the nearest millisecond.

Postprocessing of the data took place off-line by using a series of automated algorithms. R-R intervals outside of expected values were identified. Missed or spurious R-waves were flagged and corrected by linear interpolation. A 21-point moving polynomial was then applied to remove low frequency trends in the HR signal. Next, a bandpass filter extracted the variance in heart period within the frequency band of spontaneous respiration in young children $(0.24-1.04 \mathrm{~Hz})$. This process removes periodicities in the ECG signal that are outside the frequency range of the respiratory cycle. The resulting measure of RSA is in the frequency range of respiration. RSA was computed as the natural logarithm of heart period variance and reported in units of milliseconds squared and is one of several acceptable approaches for calculating RSA [28]. RSA data were calculated in 30-second overlapping windows and then averaged within each episode (baseline and attention task). The RSA data for an individual was used as long as there was a 30 -second segment with less than $20 \%$ of segments identified with artifact [29], which is the shortest duration recommended when calculating RSA with children - 30-second epochs are frequently sampled in studies of young children [30-33]. In addition, small amounts of artifact can be expected to have a minimal effect on measures of heart rate variability such as RSA [7].

Baseline RSA was assessed during a nonchallenge 'control' task. Specifically, children played with a toy alone or with an experimenter for an average of $164.8 \mathrm{~s}$ (range $=135.6-199.3$ ) while sitting at a table. Although this baseline required some attention, which may evoke vagal withdrawal [34], the toy with which the child played was simple and no task demands were placed on the child during this time.

\section{Baseline Cortisol}

Baseline cortisol was measured at age 11 because by this age the effects of early adversity and prenatal substance exposure may have become biologically embedded to impact the limbic-hypothalamic-pituitary-adrenocortical response to stress. Furthermore, the kind of problem behavior we examined in this study, including the initiation of alcohol use and risky sexual behavior, is more likely to manifest in adolescence as opposed to childhood. Baseline cortisol was collected $20 \mathrm{~min}$ before the start of a social 
stress task and involved children filling out questionnaires on topics that were innocuous and familiar to the child from previous visits (e.g. extracurricular activity, ethnic identity and nutrition questionnaires). It took approximately $20 \mathrm{~min}$ to fill out these questionnaires. We chose this task before the stress task because we wanted to control for individual differences in the type of activity the child was engaged in before coming to the laboratory and to control for differences in the car ride to the laboratory.

To collect the samples, the child deposited saliva through a straw directly into a $2-\mathrm{ml}$ vial. Ideally, the samples were $\geq 1.0 \mathrm{ml}$ but $0.5 \mathrm{ml}$ was accepted if the collection time was over $3 \mathrm{~min}$. The vials were prelabeled with study site, ID and sample type with unique barcodes (provided by Salimetrics LLC). Samples were immediately placed in a $-20^{\circ} \mathrm{C}$ freezer until shipped on dry ice to Salimetrics Laboratory [35] for assay. All samples were assayed in duplicate for salivary cortisol using a highly sensitive cortisol immunoassay kit. Each test uses $25 \mu$ lof saliva and has a limit of sensitivity of 0.007 $\mu \mathrm{g} / \mathrm{dl}$ and a range of sensitivity from 0.007 to $1.8 \mu \mathrm{g} / \mathrm{dl}$. Mean intraassay and interassay coefficients of variation were less than 5 and $10 \%$ averaged duplicate scores were used in all analyses; $97 \%$ of participants provided the prestress sample between 11:00 a.m. and 5:00 p.m. to address the diurnal cycle of cortisol that flattens between late morning and early evening. The earliest prestress sample was 10:37 a.m. and the last was 5:10 p.m. We also collected information on steroid medications, time of last meal or beverage including dairy or caffeine, and vigorous physical exercise.

The raw cortisol values (microgram/deciliter) were positively skewed and normalized using a log transformation. Outliers above or below 3 SD in all 3 samples and the difference score were winsorized by replacing the value with the value at $3 \mathrm{SD}(<1.5 \%$ of cortisol values were affected).

\section{Neurobehavioral Disinhibition}

Based on previous work $[1,18], 2$ construct scores were employed to assess neurobehavioral disinhibition at age $13 / 14$. The construct scores for behavioral dysregulation and executive dysfunction were computed from multimethod, multiagent, validated, well-established measures. The neurobehavioral disinhibition construct has good predictive validity, particularly in its ability to predict substance use onset, and good construct, discriminative and concurrent validity, as well as good test-retest and internal reliability [36].

Behavioral Dysregulation. The behavioral dysregulation construct was a composite score of 6 measures of internalizing and externalizing problems: (1) problem behaviors reported by caregivers on the Child Behavior Checklist [37] using the standardized $\mathrm{T}$ scores for total problem behaviors at age 13; (2) oppositional defiance disorder; (3) conduct disorder; (4) attention deficit disorder; (5) major depression symptom counts reported by the parent from the Diagnostic Interview Schedule for Children - IV [38] at age 14 , and (6) delinquency summary scores for the number of self-reported crimes against people and acts of general delinquency and school delinquency from the Things That You Have Done questionnaire $[39,40]$ at age 13 .

The composite was computed as the average of the 3 indicators with higher scores indicating more behavioral dysregulation. Cronbach's alpha was 0.80 for the composite score of behavioral dysregulation. Using principal components factor analyses, we obtained a single-factor solution: an eigenvalue of 3.25, explained over $50 \%$ of the variance.

Physiology, Neurobehavioral Disinhibition, Adolescent Drug Use and Risky Sex
Executive Dysfunction. This was measured with 2 computerized tests from the Cambridge Neuropsychological Test Automated Batteries [41] at age 13. There is variability in the indicators that researchers use to measure executive dysfunction as a component of neurobehavioral disinhibition. We chose tasks that assess spatial working memory, thinking time and planning, all of which have been shown to be related to brain areas related to deficiencies among children exposed prenatally to drugs [42]. The spatial working memory task is self-ordered and requires the respondent to find a 'token' in an array of colored boxes; we measured the total number of errors in the task. The Stockings of Cambridge task involves spatial planning, and the respondents must use 'balls' in one display to copy the pattern shown in another display. Thinking time includes 2 trials measuring the time to select the initial stimulus. Shorter times have been related to impulsivity. We measured the total number of correct solutions in the minimal number of moves (reverse scored to indicate executive dysfunction). The composite score was the average of the 2 indicators rescaled to $0-1$, with higher scores indicating more executive dysfunction.

\section{Substance Use}

Substance use was measured with the Things That You Have Done questionnaire yearly from ages 8 to 16 . Use of each substance (alcohol, tobacco and marijuana) was coded as yes or no (dummycoded as 1 and 0 , respectively); 3 dichotomous variables were computed based on the subject's report of use at any age: alcohol only, tobacco only or marijuana only.

Age of first sexual intercourse

Age of first sexual intercourse was reported by the adolescent at age 16 using the Adolescent Sexual Behavior Assessment [43]. The age the adolescent first engaged in any type of intercourse (vaginal or anal with the same or opposite sex) was recorded. Lower levels (compared to higher levels) of this variable indicate that children were younger when they first engaged in sexual intercourse.

\section{Statistical Analysis}

We used structural equation modeling (SEM) to test pathways between prenatal substance exposure, early adversity, physiological functioning and our behavioral, drug use and sexual behavior outcomes. All predictors were grand-mean centered and assumptions of normality were tested prior to inclusion in the model. As our interest was in the concordance between parasympathetic and neuroendocrine systems we only present SEM models in which the interaction between baseline RSA and baseline cortisol was statistically significant. Following Aiken and West [44], interactions were probed at \pm 1 SD from the mean of baseline RSA and baseline cortisol using the online-computational tools provided by Preacher et al. [45].

\section{Covariates}

Because of the diurnal rhythm of cortisol, we examined whether the time of prestress cortisol measurement was correlated with the baseline cortisol value. Time of measurement was not significantly related with the time-specific measurement of cortisol $(\mathrm{r}=$ $-0.05, \mathrm{p}=0.15)$. We also examined whether prescription and/or nonprescription steroid medication impacted cortisol concentrations. Steroid use within the last 12 hours was not significantly associated with baseline cortisol $(r=0.05, p=0.16)$. No one reported 
Fig. 1. Pathways between prenatal substance exposure, early adversity, baseline RSA, baseline cortisol, executive dysfunction, and initiation of alcohol use by age 16 among females. All pathways were modeled but only significant paths are included for ease of presentation. The dark circle represents the significant interaction between RSA and cortisol. Paths are standardized beta coefficients; $\chi^{2}(28)=67.31$, $\mathrm{p}<0.001$, CFI $=0.91$ and SMSR $=0.04$. ${ }^{*} \mathrm{p}<0.05$; $^{* *} \mathrm{p}<0.01 ;{ }^{* * *} \mathrm{p}<0.001$.

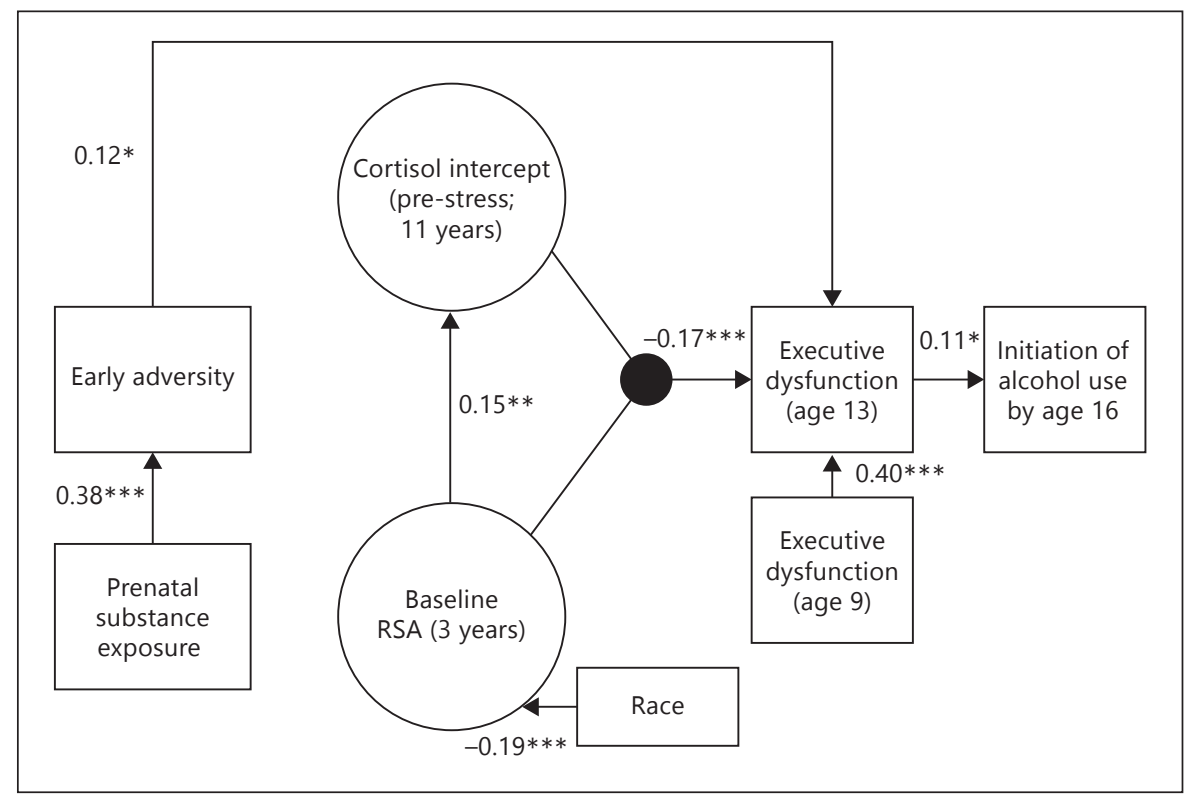

eating or drinking beverages within $1 \mathrm{~h}$ of the baseline sample or vigorous exercise within $1.75 \mathrm{~h}$ of the baseline sample.

We also examined variables that have been shown in previous studies to covary with baseline cortisol and/or RSA. These variables include child sex, race and gestational age. Race, sex and gestational age were not associated with baseline cortisol (all $\mathrm{p}>0.13$ ). Race and sex were associated with baseline RSA $(p<0.03)$ so they were included as covariates in our SEM models. We also included executive dysfunction at age 9 or behavioral dysregulation at age 9 as covariates. Because males and females may differ with respect to their drug use and risky sexual behavior, we used a multiple group SEM framework to test pathways between prenatal substance exposure, early adversity, baseline RSA, baseline cortisol, and our outcomes. This approach allowed us to examine whether the same structural model operates in different populations - in this case for males and females.

\section{Results}

At age 11, there were 860 children with cortisol data. From the original 1,388 children (assessed 11 years earlier), 388 did not participate in the visit at 11 years. A further 115 children did not participate in the cortisol reactivity task due to chronic disability $(n=57)$, child or parent unable or refusal $(n=14)$, or technical problems or resource limitations $(\mathrm{n}=44)$. Of the 885 who participated in the cortisol reactivity task, 22 had an incomplete procedure or saliva collection and 3 were excluded because the quantity of saliva was insufficient.

We analyzed whether there were differences in prenatal substance exposure or early adversity among children with and without missing cortisol data. No differences were found in exposure to substances prenatally or exposure to early adversity between children with and without cortisol data (all $\mathrm{F} \leq 2.85$, all $\mathrm{p} \geq 0.09$, all $\eta^{2} \leq 0.002$ ). Missing data were accounted for using the full information maximum likelihood feature of Mplus, which uses all available information from the observed data to provide statistically appropriate standard errors.

Of the 860 total participants, there were 41 youths who experienced no early adverse events and 178 youths without prenatal substance exposure. Both the prenatal substance exposure and early adversity variables are continuous. Inspection of data for outliers revealed that both variables were normally distributed. That the prenatal substance exposure variable was normally distributed is not surprising given that we oversampled for substance exposure in this study.

\section{Model 1: Executive Dysfunction, Drug Use, and Risky Sexual Behavior}

In the first model we examined pathways from prenatal substance exposure, early adversity, baseline cortisol and baseline RSA to executive dysfunction and drug use and risky sexual behavior outcomes. The interaction between RSA and cortisol was statistically significant in females but not in males (fig. 1). The model fit was adequate, with $\chi^{2}(28)=67.31, \mathrm{p}<0.001$, comparative fit in$\operatorname{dex}(\mathrm{CFI})=0.91$ and standardized root mean square residual $(\mathrm{SMSR})=0.04$.

Among females, greater levels of baseline RSA were related to greater baseline cortisol at age 11 . Greater ex- 
Fig. 2. Interaction between baseline RSA and baseline cortisol as a predictor of executive dysfunction in females. Simple slopes for baseline RSA and cortisol are plotted at $1 \mathrm{SD}$ above and below the mean.

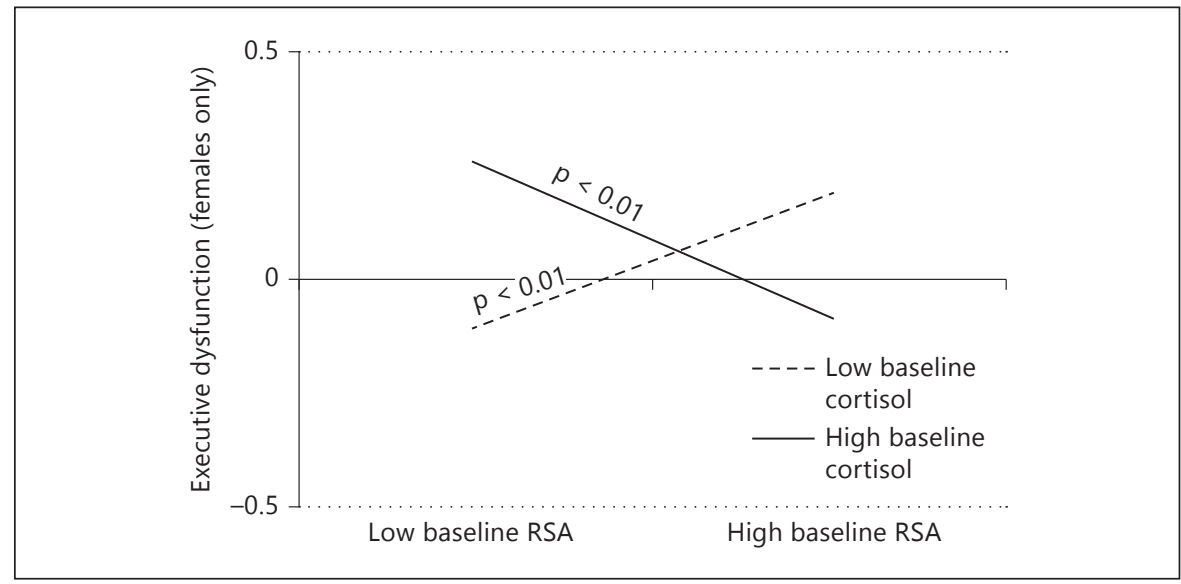

Fig. 3. Pathways between prenatal substance exposure, early adversity, baseline RSA, baseline cortisol, behavioral dysregulation, and initiation of alcohol use by age 16 among males. All pathways were modeled but only significant paths are included for ease of presentation. The dark circle represents the significant interaction between RSA and cortisol. Paths are standardized beta coefficients; $\chi^{2}(28)=73.91$, $\mathrm{p}<0.001, \mathrm{CFI}=0.92$ and SMSR $=0.05$. * $\mathrm{p}<0.05$; ** $\mathrm{p}<0.001$.

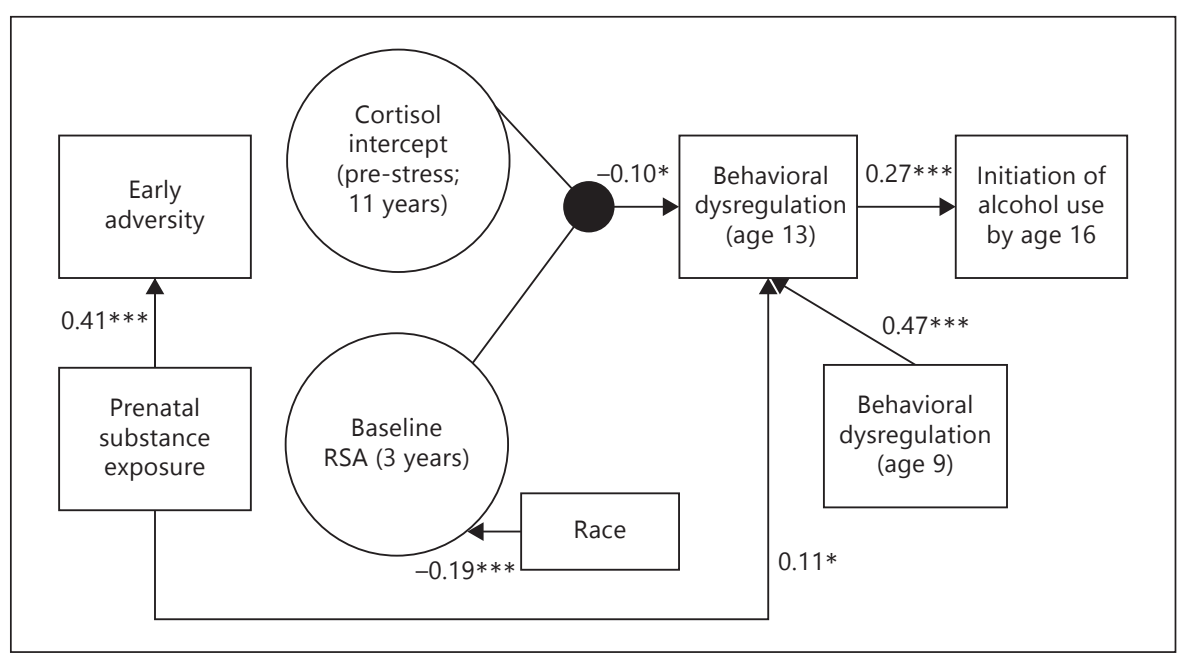

ecutive dysfunction at age 9 and greater early adversity were related to greater executive dysfunction at age 13 . The interaction between baseline RSA at age 3 and baseline cortisol at age 11 was also a significant predictor of executive dysfunction. Executive dysfunction, in turn, predicted the initiation of alcohol use by age 16 such that greater executive dysfunction indicated an increased likelihood that the child had used alcohol by age 16 . Race was also a significant predictor of baseline RSA. Post hoc tests revealed that African Americans had significantly greater baseline RSA compared to Caucasians, Hispanics and those who identified themselves as 'other'.

We probed the significant RSA and cortisol interaction at $1 \mathrm{SD}$ above and below the mean for baseline RSA and baseline cortisol (fig. 2). Executive dysfunction was lower among girls whose baseline cortisol and baseline RSA were concordant; that is, if both were high or both were low. Girls with either high baseline RSA and low baseline cortisol or low baseline RSA and high baseline cortisol had the highest levels of executive dysfunction. There were no differences in prenatal substance or early adversity exposure among these groups.

\section{Model 2: Behavioral Dysregulation, Drug Use, and Risky Sexual Behavior}

In the second model we examined pathways from prenatal substance exposure, early adversity, baseline cortisol and baseline RSA to behavioral dysregulation and drug use and risky sexual behavior outcomes. Statistically significant interactions between RSA and cortisol were only found in males.

\section{Initiation of Alcohol Use}

The model fit testing initiation of alcohol use by age 16 in the males was adequate, with $\chi^{2}(28)=73.91, \mathrm{p}<0.001$, $\mathrm{CFI}=0.92$ and SMSR $=0.05$ (fig. 3 ). There were no sig- 
Fig. 4. Interaction between baseline RSA and baseline cortisol as a predictor of behavioral dysregulation in males. Simple slopes for baseline RSA and cortisol are plotted at $1 \mathrm{SD}$ above and below the mean.

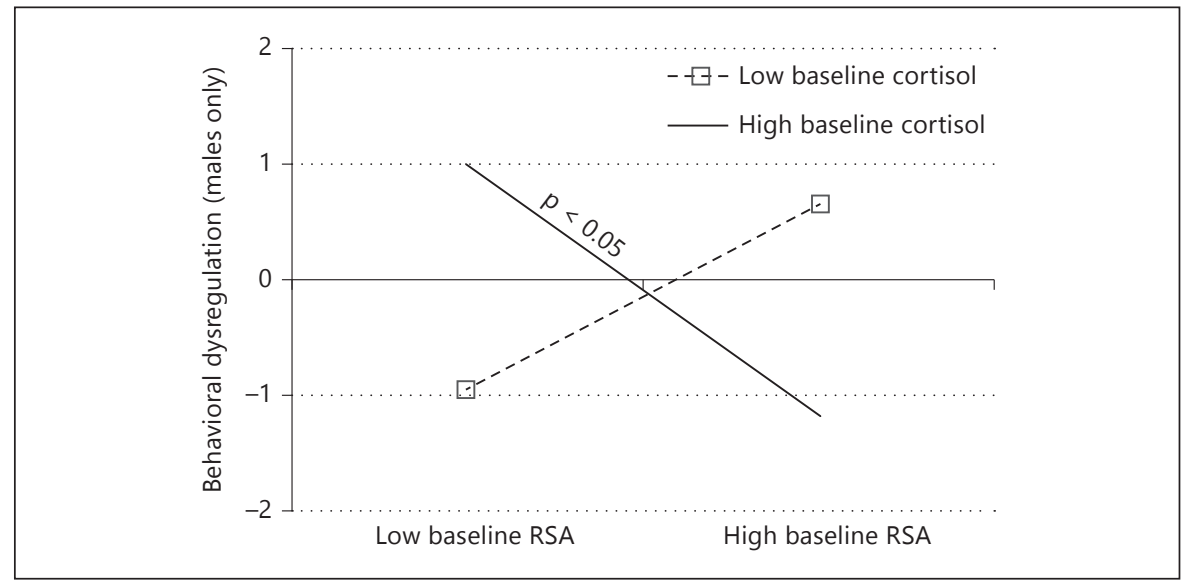

Fig. 5. Pathways between prenatal substance exposure, early adversity, baseline RSA, baseline cortisol, behavioral dysregulation, and age of first sexual intercourse among males. All pathways were modeled but only significant paths are included for ease of presentation. The dark circle represents the significant interaction between RSA and cortisol. Paths are standardized beta coefficients; $\chi^{2}(28)=62.75, p<0.001$, $\mathrm{CFI}=0.93$ and SMSR $=0.05{ }^{*} \mathrm{p}<0.05$; $* * * \mathrm{p}<0.001$.

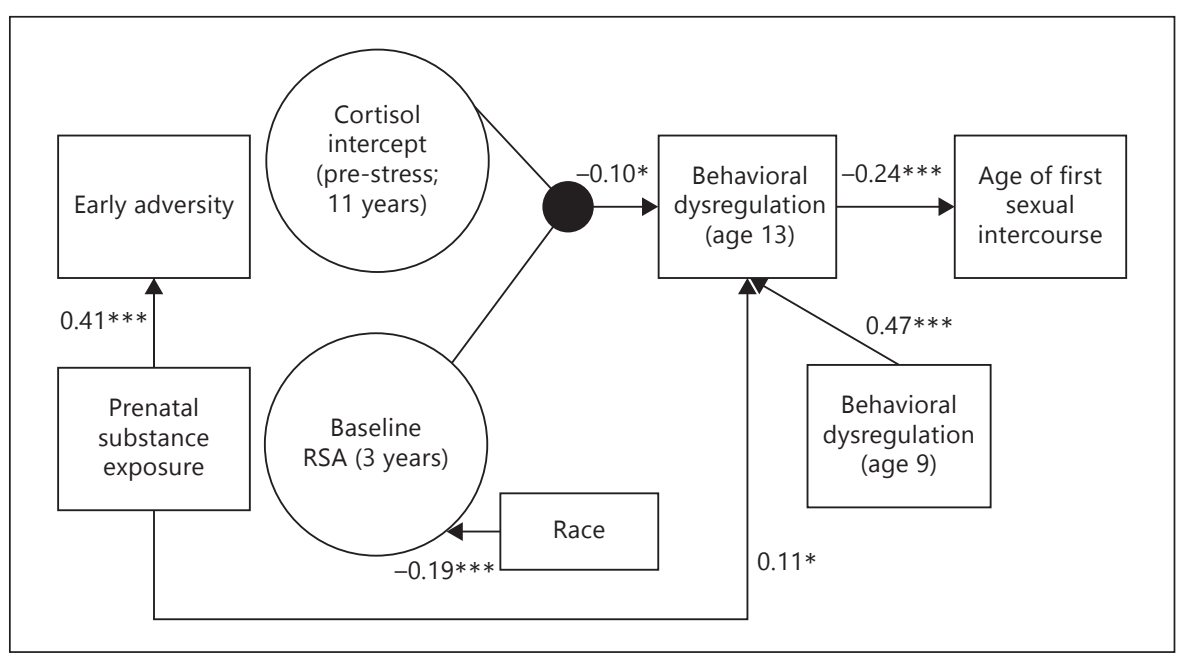

nificant predictors of baseline cortisol. Race was a significant predictor of baseline RSA at 3 years. Behavioral dysregulation at age 9 and greater prenatal drug exposure predicted greater behavioral dysregulation at age 13. Greater levels of prenatal substance exposure were associated with behavioral dysregulation at age 13. The interaction between baseline RSA and baseline cortisol predicted behavioral dysregulation at age 13. Greater behavioral dysregulation, in turn, was related to an increased likelihood that the adolescent had initiated alcohol use by age 16 .

We probed the significant RSA and cortisol interaction at $1 \mathrm{SD}$ above and below the mean for baseline RSA and baseline cortisol (fig. 4). The lowest levels of behavioral dysregulation were found among boys with both high baseline RSA and high baseline cortisol. Boys with low baseline RSA and high baseline cortisol had the highest levels of behavioral dysregulation. There were no differences in behavioral dysregulation among boys with low baseline cortisol, regardless of their baseline RSA levels. There were no differences in prenatal substance or early adversity exposure among these groups.

\section{Age of First Sexual Intercourse}

The model fit testing the pathway to age of first sexual intercourse in the males was adequate, with $\chi^{2}(28)=62.75$, $\mathrm{p}<0.001, \mathrm{CFI}=0.93$ and SMSR $=0.05$ (fig. 5). There were no significant predictors of baseline cortisol. Race emerged as a significant predictor of baseline RSA at 3 years. Behavioral dysregulation at age 9 and greater prenatal drug exposure predicted greater behavioral dysregulation at age 13. The same interaction between baseline RSA and baseline cortisol predicting behavioral dysregulation at age 13 also held for this model. Greater behavioral dysregulation, in turn, predicted a lower age of first sexual intercourse. There were no differences in prenatal substance or early adversity exposure among these groups. 


\section{Discussion}

We investigated whether physiological functioning across the parasympathetic and neuroendocrine systems would be predictive of neurobehavioral disinhibition and maladaptive outcomes in adolescence among children with prenatal substance exposure. The interaction between baseline RSA and baseline cortisol predicted unique aspects of neurobehavioral disinhibition, separately for males and females. Neurobehavioral disinhibition in turn predicted the initiation of alcohol use and age of first sexual intercourse. These results highlight the importance of studying interactions between physiological systems to predict important developmental outcomes. They also demonstrate that unique developmental pathways to these poor outcomes may exist separately for males and females.

The interaction between baseline RSA and baseline cortisol was predictive of both executive dysfunction and behavioral dysregulation, though in different ways for males and females. Females who exhibited discordance in baseline RSA and baseline cortisol (those who exhibited high levels in one system and low levels in another) had the highest levels of executive dysfunction. Higher levels of executive dysfunction in turn predicted the initiation of alcohol use by age 16 . The literature examining sex differences in executive functioning is small and equivocal, though some research suggests that planning may develop later for girls than boys [46], which may have made girls in this study more vulnerable to the initiation of alcohol use.

Discordance between baseline RSA and baseline cortisol was also found in the one other study that we know of investigating baseline effects in both the parasympathetic and neuroendocrine systems [12]. In this study, both males and females were more likely to exhibit symptoms of depression if discordant in baseline RSA and baseline cortisol. It is not surprising that children with low baseline RSA and high baseline cortisol would exhibit executive dysfunction, as this profile is reflective of poor emotional regulation and greater physiological arousal, both of which are associated with poorer performance on executive functioning tasks [47]. It is less clear why a pattern of high baseline RSA and low baseline cortisol would be associated with executive dysfunction, though it may be that discordance across systems is a stronger predictor of executive dysfunction rather than the specific pattern of parasympathetic and neuroendocrine responding. For instance, in their description of autonomic space, Berntson et al. [48] note that when the parasympathetic and

Physiology, Neurobehavioral Disinhibition, Adolescent Drug Use and Risky Sex sympathetic nervous systems are reciprocally activated (when there is activation in the parasympathetic nervous system and deactivation in the sympathetic nervous system or vice versa) the organism may be better suited to deal with survival challenges. Perhaps over the long term, consistent with the theory of allostatic load, this reciprocal activation needed to manage challenge may lead to wear and tear and subsequent 'burn-out' of these systems, which is observed functionally in this study by poorer executive function in girls. In addition, for girls only, greater early adversity was also associated with executive dysfunction. Therefore, the combination of wear and tear on both the parasympathetic and neuroendocrine systems as well as the experience of early adversity may have resulted in poorer executive functioning and subsequently the initiation of alcohol use by age 16 .

Among boys, there too existed a significant baseline RSA by baseline cortisol interaction, though in boys this interaction was predictive of behavioral dysregulation. This increase in behavioral dysregulation was in turn related to initiation of alcohol use by age 16 and lower age of first sexual intercourse. Again, the literature on sex differences in behavior control and substance use is small and equivocal. In one study, the relation between behavioral regulation (in the form of self-restraint) and substance use was stronger for boys than girls [49], though in another study this effect was stronger in girls [50]. Some literature, however, suggests that girls mature more quickly than boys in the domain of behavioral regulation, such that by age 11 it may be that the boys who have not 'caught up' with the girls are at increased risk of initiating alcohol use and engaging in risky sexual behavior. It is interesting to note that behavioral dysregulation at age 9 was associated with behavioral dysregulation at age 13 in males but not in females, suggesting that this cluster of behavioral problems emerges earlier in males, which could also reflect sex differences in maturation.

The interaction between baseline RSA and baseline cortisol was significantly predictive of behavioral dysregulation, though in a slightly different way. Male children with low baseline RSA and high baseline cortisol had the greatest levels of behavioral dysregulation while children with high baseline RSA and high baseline cortisol had the lowest levels. These findings fit with the hypothesis posed by El-Sheikh et al. [12] that poor physiological regulation in the form of low baseline RSA and greater arousal in the form of high baseline cortisol is a risk factor for poor behavioral regulation. In this study, we extend previous work as we have found that this profile of physiological responding was related to behavioral dysregulation, while 
El-Sheikh et al. found this profile was related to internalizing behavior. It appears as though high baseline RSA was protective in this sample, as high baseline cortisol in addition to high baseline RSA was related to the lowest levels of behavioral dysregulation for boys. Importantly, these results replicate those of El-Sheikh et al. [12], who found that this profile was related to the lowest levels of internalizing symptoms. In these models prenatal substance exposure was also a significant predictor of behavioral dysregulation. Prenatal substance exposure could have affected the dopaminergic reward circuitry in the brain and nucleus accumbens, affecting behavioral regulation, cognitive control and impulsivity, all of which are risk factors for the initiation of substance use and engaging in risky sexual behavior [1].

A number of limitations should be acknowledged. Although most of our physiological measures were evaluated before symptom assessments, we still cannot infer causality with these data. Future studies using a crosslagged approach will be helpful in understanding whether a physiological process at one point in time influences behavior later in development. Furthermore, it is not known whether baseline RSA and/or baseline cortisol are related to our behavioral outcomes, over and above the effects of baseline RSA and cortisol measured earlier in life. We also did not isolate the effects of a single substance, such as cocaine, on our outcomes and instead used a summary score approach, which may be a more ecologically valid indicator of prenatal substance use [51]. We acknowledge that the mechanism of action of these drugs is different, and we cannot study the relative impact of one drug over another. In addition, we chose measures of executive dysfunction which have been shown to be related to brain areas related to deficiencies among chil- dren exposed prenatally to drugs [42]. However, there may have been others (such as inhibitory control) that could have been assessed.

This study reveals sex-specific pathways to the initiation of alcohol use and risky sexual behavior through parasympathetic and neuroendocrine functioning. These results are important as they suggest that in order to better understand which adolescents are more likely to initiate substances and engage in risky sexual behavior we should assess physiological functioning across a range of systems, as opposed to studying them in isolation, as the interaction between the parasympathetic and neuroendocrine system revealed which adolescents were more likely to engage in these behaviors. Follow-up work should continue to incorporate a multiple-levels-of-analysis perspective, focusing on physiological data from multiple systems in addition to behavioral and even genetic and epigenetic information to arrive at a more complete picture of who may be at risk for the development of substance use disorders and risky sexual behavior.

\section{Acknowledgments}

This study was supported by the Eunice Kennedy Shriver National Institute of Child Health and Human Development (NICHD) Neonatal Research Network and an interinstitute agreement with the NIDA through cooperative agreements: U10DA-024117-01 and U10-HD-21385 (to S.S.), I10-DA-024128-06 and U10-HD-2786 (to H.S.B.), U10-DA-024119-01 and U10HD-27904 (to B.M.L.), U10-DA-024118-01 and U10-HD-21397 (to C.R.B.), NICHD contract N01-HD-2-3159 (to B.M.L.), and a National Research Service Award from the NIDA F32DA032175 (to E.C.). The content is solely the responsibility of the authors and does not necessarily represent the official views of the NIDA, the NICHD or the NIH.

\section{References}

-1 Lester B, et al: Neurobehavioral disinhibition predicts initiation of substance use in children with prenatal cocaine exposure. Drug Alcohol Depend 2012;126:80-86.

$>2$ Lester BM, Lagasse LL: Children of addicted women. J Addict Dis 2010;29:259-276.

-3 Granger DA, Kivlighan KT: Integrating biological, behavioral, and social levels of analysis in early child development: progress, problems, and prospects. Child Dev 2003;74: 1058-1063.

4 Beauchaine T: Vagal tone, development, and Gray's motivational theory: toward an integrated model of autonomic nervous system functioning in psychopathology. Dev Psychopathol 2001;13:183-214.
5 Obradovic J, et al: Biological sensitivity to context: the interactive effects of stress reactivity and family adversity on socioemotional behavior and school readiness. Child Dev 2010;81:270-289.

6 Shirtcliff EA, Essex MJ: Concurrent and longitudinal associations of basal and diurnal cortisol with mental health symptoms in early adolescence. Dev Psychobiol 2008;50:690-703.

7 Berntson GG, Cacioppo JT, Quigley KS: Respiratory sinus arrhythmia: autonomic origins, physiological mechanisms, and psychophysiological implications. Psychophysiology 1993;30:183-196.

8 Porges SW: The polyvagal perspective. Biol Psychol 2007;74:116-143.
9 Calkins SD, Graziano PA, Keane SP: Cardiac vagal regulation differentiates among children at risk for behavior problems. Biol Psychol 2007;74:144-153.

$>10$ Porges SW, et al: Infant regulation of the vagal 'brake' predicts child behavior problems: a psychobiological model of social behavior. Dev Psychobiol 1996;29:697-712.

11 Staton L, El-Sheikh M, Buckhalt JA: Respiratory sinus arrhythmia and cognitive functioning in children. Dev Psychobiol 2009;51:249258 .

12 El-Sheikh M, et al: Children's internalizing symptoms: the role of interactions between cortisol and respiratory sinus arrhythmia. Physiol Behav 2011;103:225-232. 
$>13$ Ulrich-Lai YM, Herman JP: Neural regulation of endocrine and autonomic stress responses. Nat Rev Neurosci 2009;10:397-409.

- 14 Thayer JF, Sternberg E: Beyond heart rate variability: vagal regulation of allostatic systems. Ann NY Acad Sci 2006;1088:361372.

15 Wallace JM Jr, et al: Gender and ethnic differences in smoking, drinking and illicit drug use among American 8th, 10th and 12th grade students, 1976-2000. Addiction 2003;98:225234.

16 Waller MW, et al: Gender differences in associations between depressive symptoms and patterns of substance use and risky sexual behavior among a nationally representative sample of US adolescents. Arch Womens Ment Health 2006;9:139-150.

17 Lester BM, et al: The Maternal Lifestyle Study: effects of substance exposure during pregnancy on neurodevelopmental outcome in 1-month-old infants. Pediatrics 2002;110 1182-1192.

18 Fisher PA, et al: The combined effects of prenatal drug exposure and early adversity on neurobehavioral disinhibition in childhood and adolescence. Dev Psychopathol 2011;23: 777-788.

19 Smith DK, et al: Child maltreatment and foster care: unpacking the effects of prenatal and postnatal parental substance use. Child Maltreat 2007;12:150-160.

20 Shankaran S, et al: Maternal Lifestyle Study: patterns of cocaine use in term pregnancy and effect on birthweight. Pediatr Res 1996;39: 279A.

21 Hollingshead A: Four Factor Index of Social Status. New Haven, Yale University Press, 1975.

22 Beck A, Steer R, Brown G: Manual for the Beck Depression Inventory - II. San Antonio, Psychological Corporation, 1996.

23 Derogatis L, Coons H: Self-Report Measures of Stress, ed 2. New York, Free Press, 1993.

24 Caldwell B, Bradley R: Home Observation for Measurement of the Environment. Little Rock, University of Arkansas at Little Rock Press, 1984

25 Sheinkopf SJ, et al: Vagal tone as a resilience factor in children with prenatal cocaine exposure. Dev Psychopathol 2007;19:649673.
26 Conradt E, Measelle J, Ablow JC: Poverty, problem behavior, and promise differential susceptibility among infants reared in poverty. Psychol Sci 2013;24:235-242.

27 MX Edit Software. Delta-Biometrics Inc, 1988-1993.

28 Berntson GG, et al: Heart rate variability: origins, methods, and interpretive caveats. Psychophysiology 1997;34:623-648.

29 Jennings JR, et al: Committee report. Publication guidelines for heart rate studies in man. Psychophysiology 1981;18:226-231.

30 Blandon AY, et al: Contributions of child's physiology and maternal behavior to children's trajectories of temperamental reactivity. Dev Psychol 2010;46:1089-1102.

- 31 Buss KA, Goldsmith HH, Davidson RJ: Cardiac reactivity is associated with changes in negative emotion in 24-month-olds. Dev Psychobiol 2005;46:118-132.

32 Moore GA, Calkins SD: Infants' vagal regulation in the still-face paradigm is related to dyadic coordination of mother-infant interaction. Dev Psychol 2004;40:1068-1080.

33 Huffman LC, et al: Infant temperament and cardiac vagal tone: assessments at twelve weeks of age. Child Dev 1998;69:624-635.

34 Suess PE, Porges SW, Plude DJ: Cardiac vagal tone and sustained attention in school-age children. Psychophysiology 1994;31:17-22.

35 Salimetrics LLC: HS Cortisol Kit Information. 2000.

36 Mezzich AC, et al: Assessment of risk for substance use disorder consequent to consumption of illegal drugs: psychometric validation of the neurobehavior disinhibition trait. Psychol Addict Behav 2007;21:508-515.

37 Achenbach TM: Manual for the Child Behavior Checklist/2-3 and 1992 Profile. Burlington, University of Vermont Department of Psychiatry, 1992.

38 Shaffer D, et al: NIMH Diagnostic Interview Schedule for Children, version IV (NIMH DISC-IV): description, differences from previous versions, and reliability of some common diagnoses. J Am Acad Child Adolesc Psychiatry 2000;39:28-38.

39 Elliott D, Huizinga D, Ageton S: Explaining Delinquency and Drug Use. Beverly Hills, Sage Publications, 1985.
40 Ingoldsby EM, et al: Conduct problems, depressive symptomatology and their co-occurring presentation in childhood as predictors of adjustment in early adolescence. J Abnorm Child Psychol 2006;34:603-621.

41 Luciana M: Practitioner review: computerized assessment of neuropsychological function in children: clinical and research applications of the Cambridge Neuropsychological Testing Automated Battery (CANTAB). J Child Psychol Psychiatry 2003;44:649-663.

42 Sheinkopf SJ, et al: Functional MRI and response inhibition in children exposed to cocaine in utero. Preliminary findings. Dev Neurosci 2009;31:159-166.

43 Mellins CA, et al: Sexual and drug use behavior in perinatally HIV-infected youth: mental health and family influences. J Am Acad Child Adolesc Psychiatry 2009;48:810-819.

44 Aiken LS, West SG: Multiple Regression: Testing and Interpreting Interactions. Newbury Park, Sage, 1991.

45 Preacher KJ, Curran PJ, Bauer DJ: Simple Intercepts, Simple Slopes, and Regions of Significance in MLR 2-Way Interactions. 2006. http://www.quantpsy.org/interact/mlr2.htm.

46 De Luca CR, et al: Normative data from the CANTAB. I. Development of executive function over the lifespan. J Clin Exp Neuropsychol 2003;25:242-254.

47 Blair C, et al: Salivary cortisol mediates effects of poverty and parenting on executive functions in early childhood. Child Dev 2011;82: 1970-1984

48 Berntson GG, Cacioppo JT, Quigley KS: Autonomic determinism: the modes of autonomic control, the doctrine of autonomic space, and the laws of autonomic constraint. Psychol Rev 1991;98:459-487.

49 Farrell AD, Danish SJ: Peer drug associations and emotional restraint: causes or consequences of adolescents' drug use? J Consult Clin Psychol 1993;61:327-334.

50 Crockett L, Raffaelli M, Shen Y: Linking selfregulation and risk proneness to risky sexual behavior: pathways through peer pressure and early substance use. J Res Adolesc 2006;16: 503-525.

51 Mayet S, et al: Drugs and pregnancy - outcomes of women engaged with a specialist perinatal outreach addictions service. Drug Alcohol Rev 2008;27:497-503. 